\title{
Anabases
}

\section{Kate Fisher, Rebecca Langlands (éd.), Sex, knowledge and receptions of the past}

Jean Zaganiaris

\section{(2) OpenEdition}

\section{Journals}

Édition électronique

URL : http://journals.openedition.org/anabases/5767

DOI : $10.4000 /$ anabases. 5767

ISSN : 2256-9421

\section{Éditeur}

E.R.A.S.M.E.

\section{Édition imprimée}

Date de publication : 10 novembre 2016

Pagination : 348-350

ISSN : 1774-4296

\section{Référence électronique}

Jean Zaganiaris, «Kate Fisher, Rebecca Langlands (éd.), Sex, knowledge and receptions of the past »,

Anabases [En ligne], 24 | 2016, mis en ligne le 15 novembre 2016, consulté le 24 septembre 2020.

URL : http://journals.openedition.org/anabases/5767 ; DOI : https://doi.org/10.4000/anabases.5767

Ce document a été généré automatiquement le 24 septembre 2020

(c) Anabases 


\title{
Kate Fisher, Rebecca Langlands (éd.), Sex, knowledge and receptions of the past
}

\author{
Jean Zaganiaris
}

\section{RÉFÉRENCE}

Kate Fisher, Rebecca Langlands (éd.), Sex, knowledge and receptions of the past, Oxford, Oxford University Press, Classical Presences, 2015.

70 livres / isbn 9780199660513

1 Comment la construction de savoirs sur la sexualité peut-elle impacter la construction de savoirs à propos du passé ? Comment les usages sociaux et intellectuels du passé influent-ils sur les représentations des pratiques sexuelles au sein d'un contexte historique donné ? C'est à cette problématisation complexe, combinant les perceptions diachroniques et synchroniques $\mathrm{du}$ passé avec une objectivation des pratiques discursives sur le sexe, que se proposent de traiter les différentes contributions. Dans leur introduction, Kate Fisher et Rebecca Langlands, les coordinatrices de l'ouvrage, partent de la problématique bien connue de Michel Foucault. La construction de savoirs peut être comprise à partir d'une approche saisissant la discontinuité des pratiques et des façons de penser. Les cadres épistémologiques diffèrent selon les périodes et les contextes, tout comme les usages du passé et les connaissances construites à partir de l'utilisation de telle ou telle source ancienne. Le rapport aux valeurs et aux normes n'est pas non plus le même et les utilisations du passé diffèrent selon la singularité des acteurs, y compris au sein d'une même période historique. Il y a des usages "idéologiques" et "stratégiques» du passé et des enjeux de légitimation chez les acteurs qui produisent des savoirs sur le sexe.

Le recours à la Grèce antique dans le monde européen des $\mathrm{XIX}^{\mathrm{e}}$ et $\mathrm{XX}^{\mathrm{e}}$ siècles a permis, par exemple, de consolider les luttes balbutiantes des mouvements gays et lesbiens. 
L'enjeu n'est dès lors pas de savoir si les interprètes auraient bien ou mal lu les textes grecs ou la poésie de Sapho mais de saisir la façon dont ils l'utilisent. La contribution de Peter Cryle consacrée aux textes libertins du XIX ${ }^{\mathrm{e}}$ siècle prend d'ailleurs pour objet d'étude les différentes formes d'anachronisme produites à partir de l'usage du passé. Cela n'empêche bien évidemment pas de contextualiser avec précision ces discours sur le sexe et leurs appropriations spécifiques des savoirs anciens afin de comprendre de quelle façon, à chaque période historique, certaines connaissances que nous jugeons aujourd'hui obsolètes étaient tenues pour vraies.

3 Les textes de Jana Funke sur Magnus Hirschfeld (l'un des premiers auteurs à plaider pour l'acceptation sociale de l'homosexualité à la fin du XIX ${ }^{\mathrm{e}}$ siècle) et de Sebastian Matzner sur Karl Heinrich Ulrichs (l'un des fondateurs de la « sexologie » au cours de la même époque) montrent que certains acteurs sont allés chercher des références au passé pour consolider leurs propres thèses. Ulrichs interprète les nombreuses références bibliographiques réunies à partir de la théorisation de la sexualité qu'il est en train de construire. Les Métamorphoses d'Ovide et la rencontre d'Hermaphroditus avec la nymphe Salmacis lui permettent d'affirmer l'existence d'un "troisième sexe " devant être théorisé à partir des études embryonnaires existant sur les hermaphrodites à la fin du XIX ${ }^{e}$ siècle. Il en est de même pour Magnus Hirscheld. Lors de ses expéditions dans les colonies, ce dernier a utilisé certaines représentations non-occidentales, notamment les cultes phalliques ornant des temples hindous, pour démontrer que les comportements homosexuels ne sont pas des actes contre-nature. Le texte de Alastair J. L. Blanchard revient sur les usages stratégiques du passé. Il montre que C. G. Chaddock, le traducteur britannique de Richard Von Krafft Ebing (un psychiatre austro-allemand célèbre), profite de ses publications pour introduire le terme « homosexuality » dans la langue anglaise à partir des années 1890. Alastair J. L. Blanchard montre également qu'un certain nombre d'auteurs ont vu dans Le Banquet de Platon, notamment dans le mythe d'Aristophane, des formes de savoirs légitimant les pratiques homosexuelles ou homo-érotiques au sein d'un monde marqué par le puritanisme victorien. C'est en ce sens que vont également les textes de Chris Water sur la postérité d'Oscar Wilde, dont l'usage des œuvres combiné à sa façon de se référer à la Grèce antique ne mèneront pourtant que tardivement à en faire un des « martyrs » de la lutte LGBT, et de Joanna de Groot évoquant la façon dont Sir Richard Burton, un officier colonial, a traduit les Mille et une nuits entre 1886 et 1888 à partir de ses propres représentations normatives du monde arabe.

4 Le passé, notamment la Grèce antique, a laissé un "héritage sans testament » aux producteurs de savoirs sur la sexualité. Dès lors, dans des univers sociaux parfois très hétéronormatifs, tels que les musées dont parle Debbie Chalis dans sa contribution, le fait de faire resurgir les différentes représentations de l'homosexualité et autres formes de sexualité non normatives venant du passé rappelle que les modes de vie minoritaires, marginalisés, infériorisés et stigmatisés font partie d'un monde commun que nous partageons tous. 


\section{AUTEURS}

JEAN ZAGANIARIS

CRESC/EGE Rabat

zaganiaris@yahoo.fr 